\section{Guanacaste, Costa Rica: a landmark for cervical cancer prevention}

\author{
Sylvia C. Robles 1 \\ and Mirta Roses Periago ${ }^{2}$
}

Every year there are 466000 new cases of invasive cervical cancer in the world, most of them in developing countries. Latin America and the Caribbean, along with Sub-Saharan Africa and Southeast Asia, exhibit some of the highest incidence rates worldwide. The incidence of cervical cancer is highest among poor women with few years of schooling, who tend to be diagnosed at advanced stages of the disease. Even when these women are screened or diagnosed, however, less than one fourth of them receive adequate follow-up and care, as shown by data from Ecuador, Paraguay, Venezuela, Peru, Bolivia, Jamaica, Nicaragua, and El Salvador (1). ${ }^{3}$

Programs for the prevention of cervical cancer have been unsuccessful in the developing world, even in countries where cytology has been available for many years and where organized health care systems exist. In industrialized countries, however, mortality from cervical cancer has shown a dramatic decrease over the past 45 years due to periodic cytologic screening. It has been difficult and expensive for many countries to develop and maintain high quality standards for cytology, to provide full access to care for women with positive cytology results, and to accomplish high coverage with cytologic screening among middle-aged women. A recent policy analysis has shown that other screening strategies may be much more cost-effective than traditional cytologic screening (Papanicolaou or Pap smear) (2). Visual inspection of the cervix with acetic acid, which provides immediate results, can assure women undelayed treatment as well. Testing for the presence of human papillomavirus (HPV) in the uterine cervix, a condition that is necessary for the development of cervical cancer, has also shown promising results in various studies.

The Pan American Health Organization, in collaboration with other partners of the Alliance for Cervical Cancer Prevention (ACCP), which is funded by the Bill and Melinda Gates Foundation, has been evaluating several prevention strategies under field conditions. Projects aimed at assessing strategies to prevent cervical cancer are under way in areas of the world where resources are few. The results of these studies will provide valuable information to decision-makers and help them to choose the most appropriate strategy in addressing their particular situation. This is especially important in order to reach women at risk in underserved areas that have not been reached before. We need novel approaches and affordable solutions that can be sustained at low cost and can prevent disease in the population at large.

Many innovations geared at preventing cervical cancer have been made possible by an increased understanding of the natural history of the disease. In the current issue of the Revista Panamericana de Salud Pública/Pan American Journal of Public Health (RPSP/PAJPH), results from the final follow-up phase of the cervical cancer study in Guanacaste, a Costa Rican province with an unusually high incidence of the disease, are presented in an article by Concepción Bratti et al. (3). The Guanacaste study was set up by the National Cancer Institute (NCI) of the United States in collaboration with Costa Rican researchers, in order to study the natural history of cervical cancer by examining the role of HPV in the development of cervical neoplasia and to assess cervical cancer screening technologies. The methods used and the preliminary results of the cohort assembled in Guanacaste were described in an article published in the RPSP/PAJPH in 1997 by Rolando Herrero (4), a Costa Rican researcher and recipient of the Fred Soper Award in Interamerican Health.

Implementation of the study in Guanacaste was a visionary step. It moved high quality research to the field, where the problem was actually occurring. We have learned a great deal from the numerous scientific publications that have emerged from the Guanacaste project over the past seven years. The NCI partnered with local researchers; in the process it built capacity and demonstrated that, if resources and technical support are made available, developing countries can effectively address local health problems and study them with scientific rigor, advance knowledge, and contribute to public health solutions. As Dr. Bratti writes in her paper,

\footnotetext{
It is possible-and in fact important-to adapt new diagnostic and screening technology invented in high-technology areas to regions of need such as rural Guanacaste. We transferred LEEP to Guanacaste early in the project. The transfer of other study
} 
technology, including visual screening, thin-layer cytology, and HPV testing, is technically complete but still too expensive for countrywide adoption, regardless of demonstrated improved accuracy. We continue to press for affordable adaptations of new cervical cancer prevention technologies. We believe that this female population was willing to participate in the study not only for their own safety but also in the hope that their efforts would guide the way toward a diminished risk of cervical cancer for the following generations. To validate a technology in an area that cannot ultimately use it (because of unavailability or cost) would be fruitless in our opinion and contrary to the cohort members' spirit of cooperation.

Dr. Bratti makes reference to the spirit of cooperation of the study population in Guanacaste. As a result of this spirit, the study had very high participation rates, and these in turn contributed to its success. The author also mentions the affordability of new effective technologies, which the public health community should clearly welcome. (Please confirm that the intended meaning has been captured.) Progress is being made in this regard by the Program for Appropriate Technology in Health (PATH), a member of the ACCP. On February 2004, PATH launched partnership with Digene Corporation, the company that produces the Hybrid Capture ${ }^{\circledR}$ test for HPV, in order to develop low-cost, easy-to-use, culturally acceptable tests for cervical cancer screening that would be suitable for areas of the world with minimal resources and poor medical infrastructure. In addition, the research partnership between Costa Rica and NCI has opened new horizons in disease prevention with the testing of a vaccine against HPV that is expected to prevent cervical cancer. New approaches to public-private partnerships that are intended to benefit key public health programs are under way. This is an area in which the Pan American Health Organization has exercised a pioneering role through its revolving fund for immunization programs in the Americas.

The lessons learned from the Guanacaste study should move other researchers from middle- and high-income countries with research capabilities to address public health problems where they occur. Building research capacity in this fashion will make it possible to advance knowledge so that public health solutions can reach those in need and health inequities can be effectively reduced through public health initiatives. The Bill and Melinda Gates Foundation has recently partnered with the National Institutes of Health and the Foundation for the National Institutes of Health (NIH Foundation) in Bethesda, Maryland, United States of America, for the study of under-researched health problems. Subsequently, the NIH Foundation launched a worldwide consultation to identify critical scientific challenges in global health and stimulate research on diseases that constitute a major burden in the developing world. It is expected that over the coming years more research will be performed in developing countries. Researchers that are given new grants to study health problems in developing countries should look carefully at the Guanacaste experience, particularly at the key factors that made this partnership a success story.

We believe that the Organization's participation in the AACP has contributed to significant advances in a number of ways, particularly the development of appropriate screening methods, their application in public health practice, technology transfer among countries, and international cooperation within the Region. It has also helped reduce disparities in access to preventive care as part of the unfinished health agenda in our hemisphere.

\section{REFERENCES}

1. Gage JC, Ferreccio C, Gonzales M, et al. Follow-up care of women with abnormal cytology in a low-resource setting. Cancer Detect Prev. 2003;27:466-471.

2. Goldie SJ, Kuhn L, Denny L, Pollack A, Wright TC. Policy analysis of cervical cancer screening strategies in low-resource settings: clinical benefits and cost-effectiveness. JAMA. 2001;285: 3107-3115.

3. Bratti MC. Description of a seven-year prospective study of human papillomavirus infection and cervical neoplasia among 10000 women in Guanacaste, Costa Rica. Rev Panam Salud Publica. 2004;15(2):75-89.

4. Herrero R, Schiffman M, Bratti C, Hildesheim A, Balmaceda I, Sherman ME, et al. Design and methods of a population-based natural history study of cervical neoplasia in a rural province of Costa Rica: the Guanacaste Project. Rev Panam Salud Publica. 1997;1(5):362375. 
This article has received corrections in agreement with the ERRATUM published in Volume 19 Number 3. 